\title{
Cost-Effectiveness of Therapeutic Drug Monitoring- Guided Adalimumab Therapy in Rheumatic Diseases: A Prospective, Pragmatic Trial
}

\author{
Catalina Gómez-Arango · Inigo Gorostiza • Eduardo Úcar · Maria Luz García-Vivar • Clara Eugenia Pérez • \\ Juan Ramon De Dios • Belen Alvarez • Ana Ruibal-Escribano • Claudia Stoye • Margarida Vasques • \\ Joaquin Belzunegui • Antonio Escobar • Ziortza Trancho • Ainhoa Ruiz del Agua • Lorena Del Rio • \\ Cristina Jorquera · Eli Diez · Antonio Martínez · Daniel Nagore
}

Received: May 28, 2021 / Accepted: July 1, 2021 / Published online: July 18, 2021

(c) The Author(s) 2021

\section{ABSTRACT}

Introduction: To assess the clinical and costeffectiveness of therapeutic drug monitoring (TDM) based on serum adalimumab levels compared to standard of care in patients with rheumatoid arthritis, psoriatic arthritis and ankylosing spondylitis.

Methods: This was a non-inferiority, multicentric, non-randomized, pragmatic trial including

Antonio Escobar is a deceased author.

Supplementary Information The online version contains supplementary material available at https:// doi.org/10.1007/s40744-021-00345-5.

C. Gómez-Arango · E. Úcar · M. L. García-Vivar .

C. E. Pérez

Department of Rheumatology, Osakidetza Basque Health Service, Basurto University Hospital, Bilbao, Spain

I. Gorostiza - A. Escobar - Z. Trancho - C. Jorquera Research Unit, Osakidetza Basque Health Service, Basurto University Hospital, Bilbao, Spain

I. Gorostiza · A. Escobar REDISSEC, Kronikgune Institute for Health Services Research, Barakaldo, Spain

J. R. De Dios · B. Alvarez - A. Ruibal-Escribano .

C. Stoye $\cdot$ M. Vasques

Department of Rheumatology, Osakidetza Basque Health Service, Araba University Hospital, Vitoria, Spain adult patients diagnosed with moderate-to-severe, clinically stable rheumatic diseases treated with adalimumab. Consecutive patients were assigned $1: 2$ to the control (CG) or the intervention group (IG), based on the site of inclusion, and followed up for 18 months. Adalimumab serum levels were measured at each study visit and released to the IG only to modify dosing strategy. Data on disease activity, healthcare resource utilization and health-related quality of life (HRQoL) measured through the EQ-5D-5L were collected. Number of persistent and overall flares, time to first flare, days experiencing high disease activity, total direct costs, quality-adjusted life years (QALYs) and incremental cost-effectiveness ratio (ICER) were calculated.
J. Belzunegui
Department of Rheumatology, Osakidetza Basque Health Service, Donostia University Hospital, San Sebastian, Spain
A. Ruiz del Agua - L. Del Rio - E. Diez · A. Martínez · D. Nagore $(\bowtie)$
R\&D Department, Progenika Biopharma S.A., A Grifols Company, 504 Parque Tecnológico Bizkaia, 48160 Derio, Spain
e-mail: daniel.nagore@progenika.grifols.com 
Results: Of the 169 recruited patients, 150 were included in the analysis (52 and 98 patients in the CG and IG, respectively). The primary endpoint was not met as persistent flares were not significantly lower in the IG, although mean (SD) number of flares was numerically lower in the IG $(0.67$ [0.70] versus 0.90 [0.82], $P=0.073)$, respectively. Based on EQ-5D-5L utilities, HRQoL was significantly higher in the IG at $3(P=0.001)$ and 6 months $(P=0.035)$, which overall translated into 0.075 QALYs gained per patient for the IG at month 18 . Overall, direct costs were significantly lower for the IG patients $(€ 15,311.59[4,870.04]$ versus $€ 17,378.46[6,556.51], P=0.030)$, resulting in the intervention being dominant, leading to increased QALY at a lower overall cost

Conclusion: Adalimumab dose tapering based on TDM for rheumatic patients led to an increased quality of life and QALY gain and entailed lower costs, being a more cost-effective alternative than clinically guided management.

Keywords: Adalimumab; Cost-effectiveness; Rheumatic diseases; Therapeutic drug monitoring

\section{Key Summary Points}

\section{Why carry out this study?}

Biological therapies, such as tumor necrosis factor (TNF) inhibitors, are safe and effective treatments for rheumatoid arthritis (RA), psoriatic arthritis (PsA) and ankylosing spondylitis (AS), although the high cost requires rational use as part of a comprehensive therapeutic strategy

Therapeutic drug monitoring (TDM) allows clinicians to inform therapeutic decisions based on serum drug levels and antibodies

The objective of this study was to assess the clinical and cost-effectiveness of TDM based on adalimumab drug levels and anti-adalimumab antibodies in clinically stable RA, PsA and AS patients who were candidates to adalimumab dose tapering

\section{What was learned from the study?}

Adalimumab dose tapering based on TDM improved health-related quality of life of rheumatic patients and reduced total direct costs, resulting in a more costeffective alternative than a clinically guided management

The current study indicates that a TDMbased strategy is economically justified and might help physicians to improve healthcare resources

\section{INTRODUCTION}

Rheumatic diseases, including rheumatoid arthritis (RA), psoriatic arthritis (PsA) and ankylosing spondylitis (AS), are chronic inflammatory conditions characterized by periods of disease activity (flares), that result in joint and systemic damage with significant impairment in quality of life [1-3]. The purpose of treatment for rheumatic diseases consists of controlling inflammatory activity, progression of structural joint and organ damage and preventing patient disability and maintaining quality of life [4].

Anti-TNF drugs (infliximab, adalimumab, etanercept, certolizumab and golimumab) have proved to be safe and effective alternatives in RA, PsA and AS. They are used as monotherapy in AS, but usually combined with methotrexate or other synthetic disease-modifying antirheumatic drugs (DMARds) in peripheral arthritis (RA and PsA). One third of patients receiving these therapies do not respond to treatment or lose initial response (secondary failure). Secondary failure may be due to sub-therapeutic serum drug levels (DLs) or antidrug antibody (ADA) formation against the biological agent, which decreases the efficacy of the biological drug and induces adverse events [5-8]. The use of biological treatments has progressively increased, resulting in an increase of healthcare expenditure and becoming a concern for policy makers. The high cost of these therapies and the limited availability of information on their 
long-term safety require rational use as part of the comprehensive therapeutic strategy [9-12].

Therapeutic drug monitoring (TDM) is a clinical decision tool that modifies dosage treatment, by measuring serum DL and ADA, to achieve drug exposure related to the maximum clinical benefit [13]. The use of TDM in rheumatic diseases, resulting in tapering adalimumab levels, is supported by the high variability in biological drug concentration among patients [14], with a direct relationship between trough adalimumab drug concentration between 5 and $8 \mu \mathrm{g} / \mathrm{ml}$ and clinical response [15]. In addition, down-titration is feasible without reducing the efficacy in stable patients. Therefore, accurate monitoring of serum drug and ADA levels should be an important part of therapy for patients treated with biological agents [14, 15].

Clinical effectiveness of TDM is achieved by reducing dosage and/or increasing the interval between doses and has successfully been implemented in patients with RA, PsA and AS $[16,17]$. Remarkably, a recent clinical trial confirms that adalimumab down-titration in RA patients in remission, with high levels of TNF blocker, is viable without increasing the rate of relapses [18]. According to current recommendations of the rheumatology and pharmacy societies, the criteria to perform dose tapering in clinical practise require that the patient stays in remission or in low disease activity (LDA) at least 6 months before dose reduction [19]. Specifically, adalimumab dose tapering consists of increasing intervals of injection from 2 to 3 weeks or even up to monthly administration [19].

Cost-effectiveness studies analysing empiric management of anti-TNF therapy against TDMbased approach evidenced that drug monitoring reduces costs in healthcare systems [20-22]. Nevertheless, there are neither clinical nor economic data on RA, PsA and AS patients, with clinically stable disease, responders to adalimumab treatment and eligible for TDM dose tapering.

With this background, we aimed to assess the effectiveness of TDM based on adalimumab serum concentration and presence of antiadalimumab antibodies in clinically stable RA,
PsA and AS patients who were candidates to adalimumab dose tapering. Health-related quality of life (HRQOL) and total direct costs were also assessed to estimate the cost-effectiveness of this intervention.

\section{METHODS}

\section{Study Design and Participants}

This was a non-inferiority, interventional, multicentre, non-randomized, pragmatic trial that included patients with moderate-to-severe RA, PsA or AS. Adult patients who were clinically stable for at least 6 months prior to inclusion (Disease Activity Score 28 [DAS28] $<3.2$ or a Bath Ankylosing Spondylitis Disease Activity Index [BASDAI] < 4) were eligible for the study. Exclusion criteria included any serious concomitant condition which prevented adequate patient follow-up.

Eligible patients were consecutively invited to participate in the study at the rheumatology departments of Basurto (Bilbao, Spain), Araba (Vitoria, Spain) and Donostia (San Sebastian, Spain) University Hospitals. Patients included at the Basurto site were assigned to the dose optimisation strategy interventional group (IG), whereas patients included at the Donostia and Araba sites were assigned to the standard of care control group (CG) in a 2:1 ratio. Patient allocation into the IG or CG was performed based on the site of inclusion because (1) sample transportation/logistics to ensure adalimumab DL and ADA were available on time for investigators to make a therapeutic decision was more convenient for the Basurto site; (2) usual clinical practice was considered equivalent among sites and investigators, and therefore no major differences were expected.

At each visit, conventional clinical practice was strictly followed according to the scientific recommendations for management and biological tapering established in clinical guidelines [19]. Given that patients attend regular visits approximately every 3 months under usual clinical practice, study follow-up visits were performed every 12 to 16 weeks. Those patients undergoing a dose-tapering strategy 
had a follow-up visit 8 weeks after the dose reduction to assess disease activity. Overall, patients were assessed up to a maximum of seven times (seven visits) for up to 18 months. Patients from both groups were tested for adalimumab DL at baseline and at each study visit, although TDM data were released only to the IG and blinded to the CG (managed according to clinical assessment only). ADA levels were also assessed at baseline and during the follow-up if adalimumab levels were undetectable or excessively low.

At inclusion, all patients were clinically stable under adalimumab treatment (40 mg). Then, treatment frequency was adjusted to every $14,21,28,35$ or 42 days based on investigator's criteria. To measure effectiveness under real-world conditions, IG investigators used DL and ADA results to modify dosing strategy based on their clinical judgement, and they were not required to follow any specific therapeutic algorithm based on TDM results. Dose-tapering was anticipated to be between 20 and $50 \%$ of the initial dose based on current recommendations [19].

Each patient signed a written informed consent form before participating in the study. The study was approved by the Basque Clinical Investigation Ethics Committee and was conducted in accordance with local regulations and with the ethical principles of the current Declaration of Helsinki.

\section{Data Collection}

Data on age, sex, disease diagnosis, duration and activity, adalimumab regimen, concomitant therapies, comorbidities, erythrocyte sedimentation rate (ESR) and C-reactive protein (CRP) were assessed for all patients.

The following clinical variables specifically associated with disease activity were collected: the DAS28 and the Health Assessment Questionnaires Disability score (HAQ-DI) were collected for RA patients; the Tender Joint Count (TJC) and the Swollen Joint Count (SJC) for PsA patients; and the BASDAI and the Bath Ankylosing Spondylitis Functional Index (BASFI) for AS patients.
For the determination of free adalimumab drug levels (trough DL) and ADA, a serum sample of $8-10 \mathrm{ml}$ was obtained up to $24 \mathrm{~h}$ before adalimumab injection and before clinical visits. DL and ADA were measured using Promonitor ${ }^{\circledR}$-ADL and Promonitor ${ }^{\circledR}$-ANTI-ADL enzyme linked immunosorbent assays (Progenika Biopharma S.A. Spain).

To assess HRQoL, patients completed the EQ5D-5L questionnaire at each study visit, which is a standardized generic measure of health status that provides a single measure to be used in clinical and cost-effectiveness analysis [23]. Patients indicated their level of impairment related to mobility, self-care, usual activities, pain/discomfort and anxiety/depression. A visual analogue scale (VAS) that ranges from 0 (the worst imaginable health state) to 100 (the best imaginable health state) is used to quantify patient's health outcome based on their perspective.

To assess healthcare resource utilization (HCRU), drug treatment costs, testing, administration and labour cost of DL and ADA kits, data on hospitalizations (number and duration), emergency room and other outpatient visits and laboratory tests and diagnostic procedure costs were collected.

\section{Outcomes}

The primary endpoint was to assess whether TDM is non-inferior compared to conventional clinical practice, measured by the cumulative incidence of patients that experienced a persistent flare. Flares were defined as a $>1.2$ increase in DAS28 score, or $>0.6$ increase if the current DAS28 score was $\geq 3.2$ from baseline, $>1$ increase in BASDAI score, or BASDAI $>4$, and were classified as persistent when lasting $>3$ months. The use of flare incidence rate, recommended as a measure of effectiveness [24], was also calculated.

Secondary endpoints were (1) total number of flares; (2) the number of days that patients experienced high disease activity; (3) time to first flare; (4) HRQoL; (5) total direct costs; (6) cost-effectiveness of the intervention based on QALY gained per incremental cost according to 
commonly used willingness to pay criteria with respect to conventional practice in Spain.

\section{Statistical Analysis}

Categorical variables were expressed as frequencies and percentages and continuous variables with normal distribution of data as mean and standard deviation (SD). The chi-square $\left(\chi^{2}\right)$ test and the Student's $t$-test or $U$-Mann-Whitney test were used for comparison of qualitative and quantitative variables, respectively. Statistical significance was set at $P<0.05$.

A non-inferiority design was used to assess the primary endpoint, since treatments had to be equally effective for the patient when determining minimal effective dose. Non-inferiority was defined in terms of the upper limit of a twosided 95\% confidence interval (CI), determined by the ratio of patients in the IG experiencing persistent flares divided by the corresponding percentage versus the CG. If the upper limit for the CI analysis was $>1.2$, non-inferiority was demonstrated. To derive the required sample size, it was assessed that $20 \%$ of patients would experience the primary outcome, with an estimated $15 \%$ of the patients with this outcome in the IG. Applying one-side testing, $\alpha=0.05$, $1-\beta=0.8$, a non-inferiority margin of $20 \%$, a 2:1 ratio (IG:CG) and considering a withdrawal rate of $10 \%$, a total of 180 patients were needed.

To assess HRQoL, utility indexes were calculated based on patient responses to the EQ-5D$5 \mathrm{~L}$ and applying the Spanish utility tariffs [25]. Utility index values range from 1 (full health state) to -1 (health state worse than death) and can be used to transform years of life gained into QALYs.

To estimate the total direct cost, the following four types of costs were considered in $€$ of 2015: drug acquisition cost (adalimumab, Humira ${ }^{\circledR} \quad 40 \mathrm{mg} / \mathrm{ml}$ (AbbVie Deutschland $\mathrm{GmbH}$, Germany) formulation calculated according to wholesale acquisition cost of $€ 1,028.29$ in Spain plus 4\% VAT minus official discount Royal Decree 8/2010), testing kit cost ( $€ 16$ for drug levels and $€ 10$ for antibody levels), hospitalizations, emergency room and other outpatient visits to specialists (considering current Spanish tariffs) and other laboratory tests and diagnostic procedures costs.

The cost-effectiveness analysis was performed from the healthcare system perspective, considering an 18-month time horizon (study follow-up), and no discount rate was applied. Incremental effectiveness was assessed in terms of QALYs gained and overall direct costs. QALYs for each patient were calculated using the area under the curve (AUC) and interpolation technique based on the utility indexes calculated from the EQ-5D-5L.

The incremental cost-effectiveness ratio (ICER) in terms of cost per QALY gained (costutility analysis) for TDM (IG) versus standard of care (CG) was calculated using the following formula:

$$
\mathrm{ICER}=\frac{\text { Cost }_{\mathrm{IG}}-\text { Cost }_{\mathrm{CG}}}{\mathrm{QALY}_{\mathrm{IG}}-\mathrm{QALY}_{\mathrm{CG}}}
$$

To assess the accuracy and robustness of our cost-effectiveness analysis, the nonparametric bootstrapping statistical technique performing 5000 replications was applied, and the 95\% CI was estimated. The bootstrapping is a statistical resampling technique that approximates the mean and variance of an estimator and builds its confidence intervals when the distribution of that particular statistic is not known.

A sensitivity analysis was conducted to assess the impact of alternative testing kit costs on the outcome of the cost-effectiveness analysis. Testing kit costs were calculated for three scenarios: drug level and antibody level determination at $€ 16 / € 10, € 25 / € 20$ and $€ 35 / € 30$, respectively. Results were expressed in terms of cost per QALYs gained. Additionally, the value of an intervention in monetary units (net monetary benefit, NMB) was calculated considering a willingness to pay (WTP) threshold of $€ 30,000$ per QALY, which is aligned with that reported by the Spanish Health Service [26], and was further assessed across a wide range of WTP thresholds, applying the following formula:

$$
\begin{aligned}
\mathrm{NMB}= & \left(\mathrm{QALY}_{\mathrm{IG}}-\mathrm{QALY}_{\mathrm{CG}}\right) \times \mathrm{WTP} \\
& -\left(\text { Cost }_{\mathrm{IG}}-\operatorname{Cost}_{\mathrm{CG}}\right)
\end{aligned}
$$




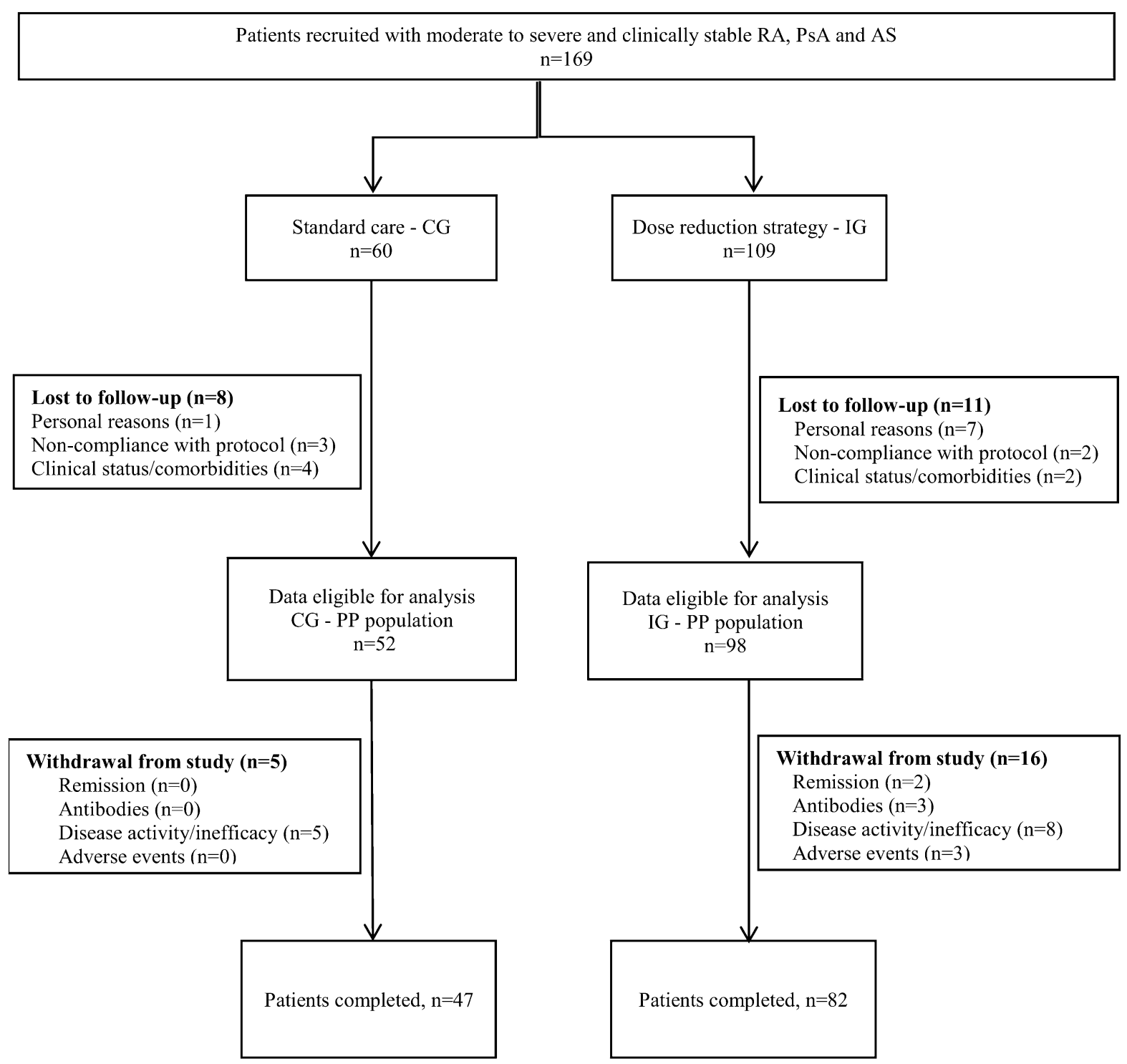

Fig. 1 Patient recruitment and follow-up flow diagram. Patients were assigned to dose reduction strategy (interventional group, IG) or standard care (control group, CG)

Positive values of the NMB would be indicative of the intervention being costeffective at that particular WTP threshold.

\section{RESULTS}

\section{Study Population}

Out of the 169 patients enrolled for study participation, 19 were lost to follow-up for personal

in a 2:1 ratio. $R A$ rheumatoid arthritis; $P s A$ psoriatic arthritis; $A S$ ankylosing spondylitis; ITT intention to treat; $P P$ per protocol

reasons, non-compliance with protocol or because of comorbidities. Final analysis of perprotocol population included 150 evaluable patients, with 52 patients grouped in the CG and 98 in the IG. During the 18 months' followup, 16 of $98(16.3 \%)$ patients in IG and 5 of 52 $(9.6 \%)$ patients in the CG withdrew from the study because of stopping adalimumab for clinical remission, low or undetectable drug levels with the presence of antibodies, inefficacy or adverse events (Fig. 1). 
Table 1 Demographic and clinical baseline patient characteristics by group

\begin{tabular}{|c|c|c|}
\hline & CG, $n=52$ & IG, $n=98$ \\
\hline \multicolumn{3}{|l|}{ General characteristics } \\
\hline Female sex, $n(\%)$ & $28(54)$ & $35(36)$ \\
\hline Age, years, mean (SD) & $53.0(13.4)$ & $53.9(11.9)$ \\
\hline \multicolumn{3}{|l|}{ Diagnostic, $n(\%)$} \\
\hline RA & $29(55.8)$ & $25(25.5)$ \\
\hline PsA & $18(34.6)$ & $30(30.6)$ \\
\hline AS & $5(9.6)$ & $43(43.9)$ \\
\hline $\begin{array}{c}\text { Disease duration, } \\
\text { months (SD) }\end{array}$ & $129.7(86.2)$ & $152.0(100.1)$ \\
\hline \multicolumn{3}{|l|}{$\begin{array}{l}\text { Concomitant treatment, } \\
n(\%)\end{array}$} \\
\hline Methotrexate & $32(61.5)$ & $31(31.6)$ \\
\hline Leflunomide & $5(9.6)$ & $7(7.1)$ \\
\hline Salazopyrin & $0(0)$ & $3(3.1)$ \\
\hline Hydroxychloroquine & $3(5.8)$ & $3(3.1)$ \\
\hline \multicolumn{3}{|l|}{$\begin{array}{l}\text { Adalimumab dose } \\
\text { intervals, } n(\%)\end{array}$} \\
\hline $40 \mathrm{mg} / 14$ days & $40(76.9)$ & $51(50)$ \\
\hline $40 \mathrm{mg} / 21$ days & $7(13.5)$ & $35(35.7)$ \\
\hline $40 \mathrm{mg} / 28$ days & $3(5.8)$ & $12(12.2)$ \\
\hline $40 \mathrm{mg} / 35$ days & $0(0)$ & $1(1.0)$ \\
\hline $40 \mathrm{mg} / 42$ days & $2(3.8)$ & $0(0)$ \\
\hline $\begin{array}{l}\text { Adalimumab dose/day, } \\
\text { mg (SD) }\end{array}$ & $2.57(0.55)$ & $2.33(0.57)$ \\
\hline \multicolumn{3}{|l|}{ Comorbidities, $n$ (\%) } \\
\hline Hypertension & $13(25.0)$ & $32(32.7)$ \\
\hline Diabetes & $4(7.7)$ & $8(8.2)$ \\
\hline Hypercholesterolemia & $13(25.0)$ & $32(32.7)$ \\
\hline Dyslipidaemia & $8(15.4)$ & $12(12.2)$ \\
\hline Hyperuricemia & $4(7.7)$ & $7(7.1)$ \\
\hline
\end{tabular}

$C G$ control group; $I G$ intervention group; $S D$ standard deviation; $R A$ rheumatoid arthritis; $P S A$ psoriatic arthritis; $A S$ ankylosing spondylitis

\section{Patient Characteristics and Disease Activity at Baseline}

Baseline patient characteristics are shown in Table 1. Overall, 28 (54\%) and 35 (36\%) patients included in the IG and CG, respectively, were female, and mean (SD) age was 53.0 (13.4) and 53.9 (11.9) years. Mean age was not different among the RA, PsA and AS sub-groups. The patient distribution based on the initial diagnosis was not completely balanced between groups, with a higher presence of RA patients in the CG than in the IG, 29 (55.8\%) versus 25 $(22.5 \%)$, respectively, and a lower proportion of AS patients, 5 (9.6\%) versus 43 (43.9\%). The most common adalimumab dose interval was every 14 days, and methotrexate was the main concomitant treatment. The presence of comorbidities was well balanced between groups.

At baseline, 9 (17.3\%) and 28 (28.6\%) patients had LDA and $43(82.7 \%)$ and 70 (71.4\%) patients were in remission in the CG and IG, respectively. No significant differences in ESR and CRP levels were observed between groups. Mean (SD) adalimumab DLs were 6.31 (3.81) and $5.53(3.48) \mathrm{mg} / \mathrm{l}$ in the CG and IG, respectively $(p=0.430)$ (Table 2$)$.

\section{Evaluation of TDM-Based Strategy During Follow-Up Period}

Patients included in the CG and IG were followed up for 544.6 (122.9) and 530.8 (104.9) mean (SD) days, respectively. No significant differences were observed in the number of days in remission or low activity (475.6 [176.5] vs. 461.0 [155.8]) or in high activity days (47.15 [96.95] vs. 45.28 [91.44]) between patients from the CG and IG, respectively. Patients continued their concomitant treatment with DMARDs during the follow-up period.

Regarding the non-inferiority requirement between both treatment groups, the number of patients with persistent flares was compared. The percentage of patients experiencing at least one persistent flare was higher in the IG compared to the CG $(28.6 \%$ vs. $23.1 \%, P=0.469 ; \mathrm{RR}$ 1.238 [95\% CI 0.689-2.226]), respectively. To 
Table 2 Disease activity level, markers and adalimumab levels at baseline by group

CG

All patients, $n=150, n(\%)$

$52(34.7)$

\section{IG}

$98(65.3)$

Disease activity level

LDA, $n(\%)$

Remission, $n$ (\%)

Days in remission, mean (SD)

RA cohort, $n=54, n$ (\%)

Disease duration, months, mean (SD)

Disease activity

$$
\text { LDA, } n \text { (\%) }
$$

Remission, $n$ (\%)

Dose ADL/day

HAQ-DI, mean (SD)

DAS28, mean (SD)

PsA cohort, $n=48, n$ (\%)

Disease duration, months, mean $(\mathrm{SD})$

Disease activity

$$
\text { LDA, } n \text { (\%) }
$$

Remission, $n$ (\%)

Dose ADL/day

TJC, mean $(\mathrm{SD})^{\mathrm{a}}$

SJC, mean $(\mathrm{SD})^{\mathrm{b}}$

AS cohort, $n=48, n$ (\%)

Disease duration, months, mean (SD)

Disease activity

LDA, $n$ (\%)

Remission, $n$ (\%)

Dose ADL/day

BASDAI, mean (SD)

BASFI, mean (SD)

Markers

CRP, mg/dl, mean (SD)

ESR, $\mathrm{mm} / \mathrm{h}$, mean $(\mathrm{SD})$

Adalimumab levels, $\mu \mathrm{g} / \mathrm{ml}$, mean $(\mathrm{SD})^{\mathrm{c}}$
$9(17.3)$

43 (82.7)

$536.3(336.2)$

$29(53.7)$

$118.3(66.3)$

$$
8 \text { (27.6) }
$$

$21(72.4)$

$15(3.1)$

$0.53(0.57)$

$2.30(0.71)$

18 (37.5)

$154.9(113.6)$

$$
\begin{gathered}
1(5.6) \\
17(94.4)
\end{gathered}
$$

$18.3(7.6)$

$0.29(0.77)$

$0.21(0.55)$

$5(10.4)$

104.6 (68.1)

$$
\begin{gathered}
0(0) \\
5(100) \\
22.4(11.5) \\
3.34(2.26) \\
2.48(2.55)
\end{gathered}
$$

0.44 (1.33)

$13.42(11.63)$

$5.82(4.04)$

$\begin{aligned} 28(28.6) & 0.164 \\ 70(71.4) & - \\ 472.6(447.7) & 0.375 \\ 25(46.2) & - \\ 183.8(88.4) & 0.003\end{aligned}$

$$
11(44)
$$

0.208

$14(56)$

$18.2(6.4)$

0.019

$0.67(0.56)$

0.357

$2.48(0.60)$

0.294

$30(62.5)$

$-$

$102.3(58.0)$

0.039

$$
8 \text { (26.7) }
$$

0.125

$22(73.3)$

-

$19.4(4.8)$

0.545

$0.12(0.50)$

0.307

$0.28(0.66)$

0.594

$43(89.6)$

$-$

$168.3(117.1)$

0.242

$$
9 \text { (20.9) }
$$

0.596

$34(79.1)$

$-$

$17.9(4.7)$

0.096

2.25 (1.93)

0.247

2.29 (1.84)

0.835

$0.36(0.36)$

0.569

13.91 (13.32)

0.826

$5.31(3.59)$

0.430 
Table 2 continued

\begin{tabular}{|c|c|c|c|}
\hline & CG & IG & $P$-value \\
\hline$<5 \mu \mathrm{g} / \mathrm{ml}, n(\%)$ & $24(47.1)$ & $49(50.0)$ & - \\
\hline $5-8 \mu \mathrm{g} / \mathrm{ml}, n(\%)$ & $12(23.5)$ & $26(26.5)$ & - \\
\hline$>8 \mu \mathrm{g} / \mathrm{ml}, n(\%)$ & $15(29.4)$ & $23(23.5)$ & - \\
\hline Anti-ADL levels, AU/ml, mean $(\mathrm{SD})^{\mathrm{d}}$ & $776.3(605.2)$ & $636.0(498.5)$ & 0.749 \\
\hline
\end{tabular}

$C G$ control group; $I G$ interventional group; $S D$ standard deviation; $L D A$ low disease activity; $R A$ rheumatoid arthritis; $P_{s} A$ psoriatic arthritis; $A S$ ankylosing spondylitis; $A D L$ adalimumab; $H A Q-D I$ Health Assessment Questionnaire-disability score; DAS28 Disease Activity Score-28; TJC tender joint count; SJC swollen joint count; BASDAI Bath Ankylosing Spondylitis Disease Activity Index; BASFI Bath Ankylosing Spondylitis Functional Index; ESR erythrocyte sedimentation rate

${ }^{\text {a }}$ Baseline TJC values were available for $n=17$ (CG) and $n=42$ (IG)

b Baseline SJC values were available for $n=47$ (CG) and $n=54$ (IG)

c Adalimumab baseline levels were available for $n=51$ (CG) and $n=98$ (IG). Patients with adalimumab level $<5 \mu \mathrm{g} / \mathrm{ml}$ included $n=8$ with undetectable levels due to the presence of anti-ADL antibodies

d Anti-ADL antibodies were detected in $n=3$ (CG) and $n=3$ (IG)

further assess the clinical effectiveness of TDM, mean number of flares as a measure of disease activity was assessed after the 18-month followup (Table 3). Mean number of flares in the IG (0.67 [0.70]) was numerically lower compared to the CG $(0.90[0.82]), \quad(P=0.073)$. Similarly, results of risk to first flare survival analysis evidenced that, although the survival function was not significantly different between groups $(P=0.302)$, median time to first flare was lower for the CG compared to the IG (136.5 days versus 145 days), and the cumulative incidence was higher (0.63 flares/patient-year versus 0.48 flares/patient-year; incidence rate ratio [IRR] = 0.77 [0.53-1.11]).

Results of adalimumab dose tapering are shown in Table 3. Overall, dosing intervals were increased in $18(34.6 \%)$ and 35 (35.7\%) patients included in the CG and IG, respectively. On average, adalimumab doses were tapered similarly in both groups, although the number of patients with a single tapering was numerically higher in the IG $(22.4 \%)$ compared to the CG (11.5\%). Adalimumab serum concentration was numerically lower for the IG group compared to the CG group through all the study visits. Adalimumab treatment was stopped in those patients with low DL and those with positive ADA formation.

\section{Quality of Life}

There were no significant differences between groups, on either the EQ-5D-5L utility index (Fig. 2a) or the VAS index (Fig. 2b), at baseline. EQ-5D-5L index remained relatively stable over time with utility values that were slightly numerically higher for the IG compared to the CG through all the study visits. These numerical differences reached statistical significance at month 3 (visit 2, $P=0.001$ ) and 6 (visit 3 , $P=0.035$ ) (Fig. 2).

\section{Cost-Effectiveness Analysis of the Intervention}

To assess whether DL and ADA determination is a cost-effective intervention to inform therapeutic decisions (vs. standard of care), HCRU, total direct costs incurred and QALYs gained during the 18-month follow-up were estimated (Table 4). The number of DL tests performed per group was similar but determinations in the CG were only informative and released to investigators only after study completion. Overall mean (SD) direct costs per patient were significantly lower $(P=0.030)$ for the IG than the CG. The amount of adalimumab used was higher for the CG. Consequently, mean (SD) cost of 
Table 3 Disease activity level, flares and adalimumab dosing interval increase during 18 months of follow-up by group

\begin{tabular}{|c|c|c|c|}
\hline & CG, $n=52$ & IG, $n=98$ & $P$-value \\
\hline Duration of follow-up, days, mean (SD) & $544.6(122.9)$ & $530.8(104.9)$ & 0.473 \\
\hline \multicolumn{4}{|l|}{ Disease activity, days, mean (SD) } \\
\hline Low activity or remission & $475.2(176.53)$ & $467.0(155.78)$ & 0.770 \\
\hline High activity & $47.15(96.95)$ & $45.28(91.44)$ & 0.907 \\
\hline \multicolumn{4}{|l|}{ Flares } \\
\hline Number of patients with a persistent flare, $n(\%)$ & $12(23.08)$ & $28(28.57)$ & 0.469 \\
\hline Number of flares per patient, mean (SD) & $0.90(0.82)$ & $0.67(0.70)$ & 0.073 \\
\hline Time to first flare, days, median (IQR) & $136.5(111.25-300.0)$ & $145.0(112.75-300.0)$ & 0.364 \\
\hline \multicolumn{4}{|l|}{ Number of patients with dosing interval increase, $n$ (\%) } \\
\hline 0 & $34(65.4)$ & $63(64.3)$ & - \\
\hline 1 & $6(11.5)$ & $22(22.4)$ & - \\
\hline 2 & $10(19.2)$ & $9(9.2)$ & - \\
\hline 3 & $2(3.8)$ & $3(3.1)$ & - \\
\hline 4 & $0(0)$ & $1(1.0)$ & - \\
\hline Total & $52(34.7)$ & $98(65.3)$ & - \\
\hline \multicolumn{4}{|l|}{ Adalimumab levels ${ }^{a}, \mu \mathrm{g} / \mathrm{ml}$, mean (SD) } \\
\hline Visit $2(50 / 93)$ & $6.31(3.82)$ & $5.51(3.86)$ & 0.238 \\
\hline Visit $3(47 / 92)$ & $6.18(4.02)$ & $5.79(3.76)$ & 0.574 \\
\hline Visit $4(48 / 87)$ & $6.47(3.98)$ & $5.80(3.84)$ & 0.342 \\
\hline Visit $5(47 / 86)$ & $6.36(4.29)$ & $5.48(3.54)$ & 0.206 \\
\hline Visit $6(36 / 73)$ & $5.90(3.72)$ & $5.57(3.40)$ & 0.642 \\
\hline Visit $7(12 / 23)$ & $5.28(4.04)$ & $4.95(3.60)$ & 0.700 \\
\hline
\end{tabular}

$C G$ control group; $I G$ intervention group; $S D$ standard deviation; $T D M$ therapeutic drug monitoring

${ }^{a}$ Patients $(n)$ with available adalimumab levels per visit and group are shown as (CG/IG)

adalimumab per patient was also higher for the CG, $€ 15,910.67$ (5179.67), than the IG, $€ 14,334.09$ (4555.23); $P=0.022$. Mean (SD) QALYs gained per patient during the 18-month follow-up were $1.086(0.411)$ and $1.161(0.326)$ for the in the CG and IG, respectively. Therefore, the intervention would be dominant, as it resulted in a 0.0855 increase in QALYs per patient but incurred lower costs $(€-2067)$. To assess the robustness of these figures, a bootstrap analysis was performed; Fig. 3a exhibits the scatterplot ICER analysis, which includes 5000 bootstrap replications for total costs, and $97.5 \%$ of them fell in the more effective and less costly quadrant of the cost-effectiveness plane (southeast quadrant). The lower and upper limits of the $95 \%$ CIs based on the percentile method were $€-255,870$ and $€ 249,031$, respectively.

To extrapolate cost-effectiveness analysis among different healthcare systems, a sensitivity analysis was conducted based on three 


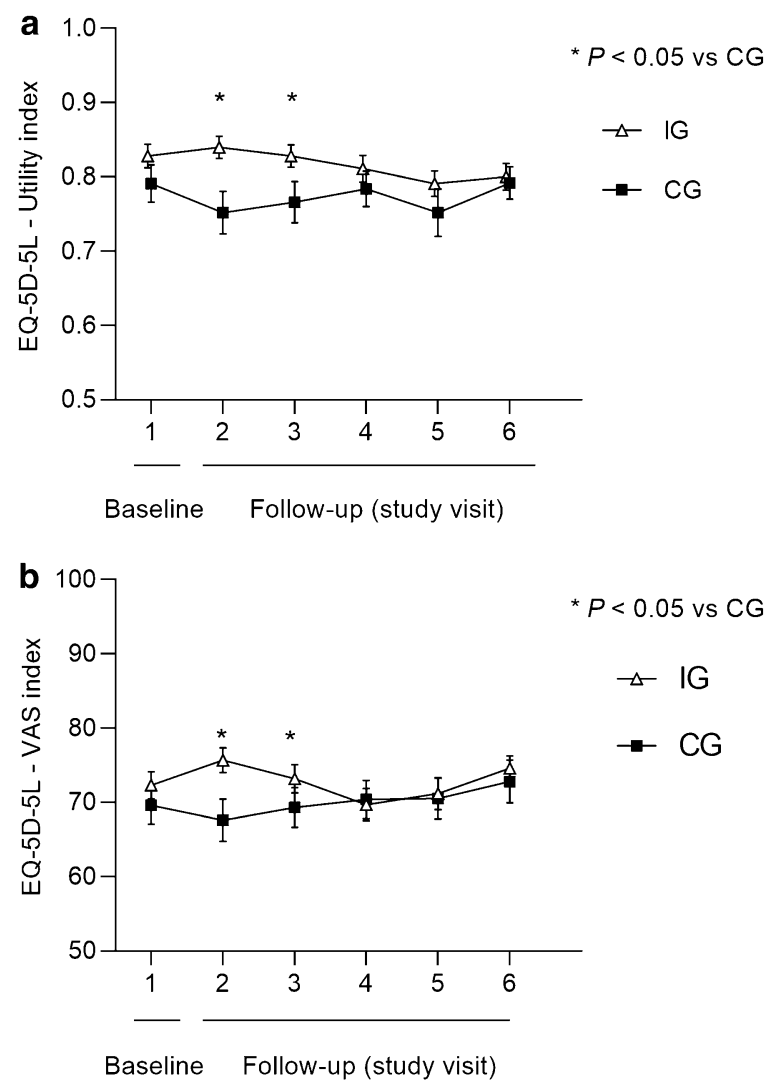

Fig. 2 Health-related quality of life (HRQoL). Change in EQ-5D-5L quality of life. a Utility index and $\mathbf{b}$ VAS index for 18 months' follow-up. Patients were visited every 3 months according to their current clinical status, although the number of completed visits was not homogeneous. Number of patients per visit in the control group (CG) and in the intervention group (IG) were at baseline $(n=52 ; n=98)$, visit $1 \quad(n=51 ; n=98)$, visit 2 $(n=48 ; n=94)$, visit $3(n=48 ; n=94)$, visit 4 $(n=48 ; n=85)$, visit $5(n=42 ; n=84)$ and visit 6 $(n=35 ; n=74)$. Data are expressed as mean $\pm 1.96^{*}$ SEM. ${ }^{*} P<0.05$ (IG versus CG). The frequency of visits was between 12 and 16 weeks and followed the clinical practice of each site. The number of visits completed per patient was not homogeneous

testing kit costs. In all evaluated scenarios, the intervention was dominant, with an increase of 0.085 in QALYs (Supplementary Table S1), supporting the results from the main cost-effectiveness analysis.

Finally, the NMB point estimate was calculated for a WTP of $€ 30,000 / \mathrm{QALY}$ resulting in $€ 4,631.87$. The NMB graphic representation for the bootstrap replications at different WTPs evidenced that DL and ADA to inform therapeutic decisions (vs. standard of care) is a costeffective (positive) intervention in all WTP scenarios. However, an imprecision is observed when the WTP increases; the lower limit of the $95 \%$ CI cuts the $X$ axis close to $€ 64,777$ (Fig. 3b).

\section{DISCUSSION}

TDM-guided management of patients treated with biological therapies is a rational personalised treatment strategy to optimise dosing regimens in rheumatic diseases, to avoid dosedependent adverse events and to increase treatment efficiency by keeping the minimal effective dose that allows the patient to stay in LDA as long as possible [16]. In this study, we evaluated the effect of TDM, based on adalimumab DL determination, to inform therapeutic decisions in clinically stable rheumatic patients under LDA or remission.

This therapeutic strategy is based on reducing the number of subcutaneous injections by progressively spacing out the interval between them rather than decreasing the treatment dose. Our data showed that, although the noninferiority primary endpoint of total persistent flares was not met, TDM in routine clinical practice could allow effective dose tapering in RA, PsA and AS patients with sub-therapeutic serum drug levels without an increase in the overall flares. The total number of flares per patient was numerically lower in the IG than in the CG, which may indicate that monitoring adalimumab DL to inform therapeutic decisions could lead to successful tapering strategies. Our data show that, although the number of flares was lower in the IG, once the flare occurs, it may be more persistent. Hence, we could hypothesize that a potential benefit of adalimumab tapering would be a reduction of adverse events, since some of them are considered dose related.

It is important to highlight that the current study has included patients responding to adalimumab therapy, mainly in remission, and that adalimumab DLs were close to $5 \mu \mathrm{g} / \mathrm{ml}$. Additionally, at baseline, patients were already 
Table 4 Expected health care resources utilization, costs and QALYs over 18-month follow-up for each treatment group

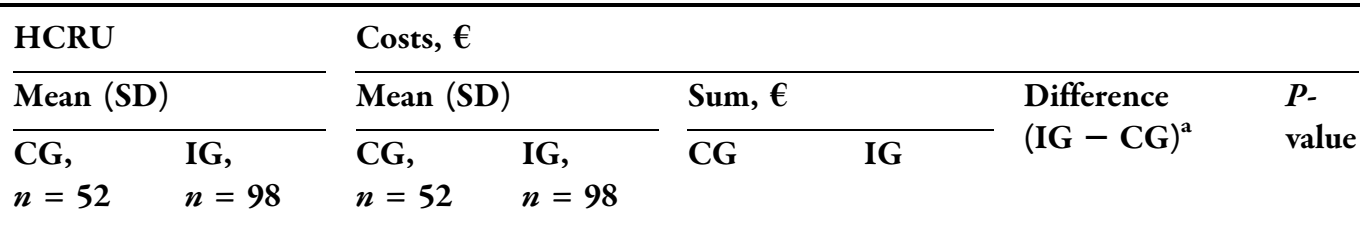

\begin{tabular}{|c|c|c|c|c|c|c|c|c|}
\hline \multicolumn{9}{|l|}{ Drug, mg } \\
\hline $\begin{array}{l}\text { Adalimumab }^{\mathrm{b}} \text {, } \\
\mathrm{mg}\end{array}$ & $\begin{array}{l}1287.6 \\
(419.5)\end{array}$ & $\begin{array}{l}1160.7 \\
(369.6)\end{array}$ & $\begin{array}{l}15,911 \\
\quad(5180)\end{array}$ & $\begin{array}{l}14,334 \\
(4555)\end{array}$ & $827,354.70$ & $1,404,741$ & -1577 & 0.056 \\
\hline \multicolumn{9}{|l|}{$\begin{array}{l}\text { Healthcare } \\
\text { resources }\end{array}$} \\
\hline Hospitalizations & $\begin{array}{l}0.12 \\
\quad(0.38)\end{array}$ & $\begin{array}{l}0.08 \\
\quad(0.34)\end{array}$ & $\begin{array}{l}825 \\
\quad(3668)\end{array}$ & $\begin{array}{l}238 \\
\quad(1106)\end{array}$ & 42,883 & 23,297 & -587 & 0.145 \\
\hline Visits to ICU & $\begin{array}{l}0.40 \\
(0.77)\end{array}$ & $\begin{array}{l}0.40 \\
(1.23)\end{array}$ & $60(115)$ & $59(182)$ & 3129 & 5811 & -1 & 0.975 \\
\hline Medical visits & $\begin{array}{l}5.81 \\
(1.22)\end{array}$ & $\begin{array}{l}5.76 \\
(1.24)\end{array}$ & $575(121)$ & $570(123)$ & 29,898 & 55,836 & -5 & 0.805 \\
\hline \multicolumn{9}{|l|}{ Testing } \\
\hline TDM kits ${ }^{c}$ & $5.6(1.4)$ & $5.6(1.4)$ & $\mathrm{n} / \mathrm{a}$ & $\begin{array}{l}91.71 \\
\quad(19.54)\end{array}$ & $\mathrm{n} / \mathrm{a}$ & 8988 & 91.71 & $\mathrm{n} / \mathrm{a}$ \\
\hline Other lab tests & - & - & $8(24)$ & $19(33)$ & 415 & 1863 & 11 & 0.035 \\
\hline \multicolumn{9}{|l|}{ Total $^{\mathrm{d}}$} \\
\hline $\begin{array}{l}\text { Without } \\
\text { Hospitalization }\end{array}$ & - & - & $\begin{array}{l}16,554 \\
(5218)\end{array}$ & $\begin{array}{l}15,074 \\
(4635)\end{array}$ & 860,796 & $1,477,239$ & -1480 & 0.077 \\
\hline $\begin{array}{l}\text { With } \\
\text { Hospitalization }\end{array}$ & - & - & $\begin{array}{l}17,378 \\
(6557)\end{array}$ & $\begin{array}{l}15,312 \\
(4870)\end{array}$ & 903,679 & $1,500,536$ & -2067 & 0.030 \\
\hline QALYs, (SD) & - & - & $\begin{array}{l}1.076 \\
(0.411)\end{array}$ & $\begin{array}{l}1.161 \\
(0.326)\end{array}$ & - & - & 0.085 & 0.224 \\
\hline
\end{tabular}

$H C R U$ health care resources utilization; $C G$ control group; $I G$ intervention group; $S D$ standard deviation; $Q A L Y$ qualityadjusted life year; TDM therapeutic drug monitoring

a IG-CG difference: the mean difference in QALY or costs between the TDM-guided strategy and control group

b Adalimumab indicates the mean quantity (mg) used during the 18-month follow-up according to the regimen prescribed to patients. The cost $(€)$ was obtained multiplying the time in days which the patient was undergoing treatment according to a specific regimen and the cost of Humira ${ }^{\circledR}$

${ }^{c}$ Only nine patients $(9.2 \%)$ of the IG and five $(9.6 \%)$ of the CG required anti-ADL antibody determinations during follow-up. n/a: Cost of Promonitor ${ }^{\circledR}-A D L$ and Promonitor ${ }^{\circledR}$-anti-ADL tests were $€ 16 / € 10$, respectively. n/a: not applicable since costs of TDM tests were only calculated for the IG

${ }^{d}$ Total $(€)$ indicates overall study cost during the 18 months follow-up

receiving tapered dosing regimens, and with adalimumab drug levels closer to $5 \mu \mathrm{g} / \mathrm{ml}$ in the interventional group, suggesting that the opportunity to improve tapering was smaller in this group. This may have underestimated the benefits of TDM in a group of patients under 

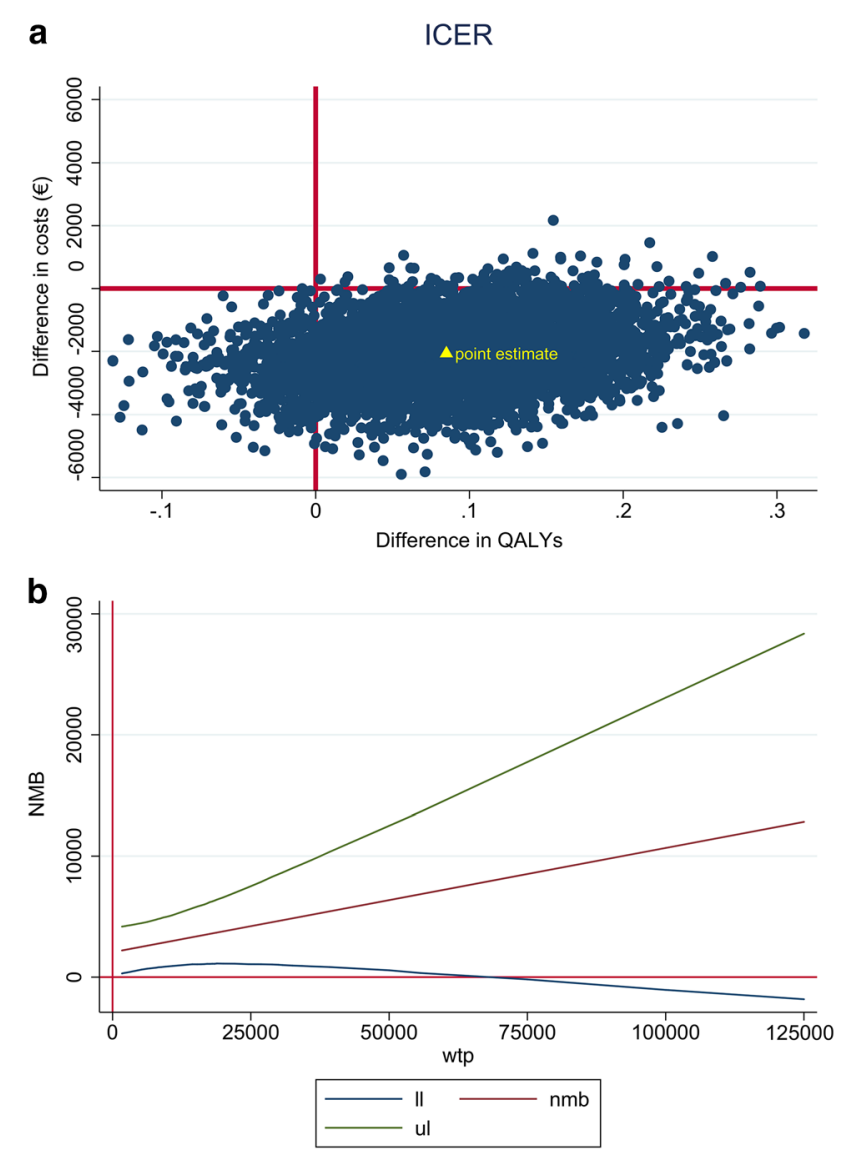

Fig. 3 Cost-effectiveness analysis. a Incremental costeffective ratio (ICER) analysis, including 5000 bootstrapping replications per group for the comparison of IG versus CG during the 18-month follow-up. QALY qualityadjusted life years. b Mean incremental net monetary

standard dosing. Previous studies have confirmed that adalimumab DLs between 5 and $8 \mu \mathrm{g} / \mathrm{ml}$ are related to higher rates of clinical response $[15,27]$ and patients with high DLs ( $>8 \mu \mathrm{g} / \mathrm{ml}$ ) were related to lower rates of relapses after dose reducing strategy [18] and could safely extend the dosing interval [28]. Our data also suggest that measuring DL proactively prior to each tapering would be needed to identify appropriate candidates for TDM and to avoid those with low adalimumab DLs. Interestingly, those patients who stopped adalimumab treatment because of very low or undetectable DL or presence of ADA were from the IG, suggesting that TDM would be of the utmost importance to stop unnecessary drug treatment in patients in benefit (NMB) for the bootstrap replications at different WTPs with 95\% confidence intervals evidencing that measuring DL to inform therapeutic decisions is a costeffective (positive) intervention. WTP willingness to pay; $l l$ lower limit; $u l$ upper limit

remission. In the current study, clinical effectiveness of TDM was assessed for 18 months. A previous observational study suggested that biological therapies are safe and effective for patients with arthritis, although long-term clinical remission after 24 months was not frequent [29].

It is important to highlight that, although the number of patients experiencing a persistent flare during the study follow-up was numerically higher in the IG, this did not translate into a worsened HRQoL or increased HCRU. In fact, EQ-5D-5L utility index was numerically higher for the IG, indicating better HRQoL, through all the study visits and significantly higher 3 and 6 months after baseline. 
Likewise, hospitalizations, emergency room visits and other outpatient visits were numerically lower in the IG.

In our cost-effectiveness analysis, the use of DL and ADA to inform therapeutic decisions on adalimumab dose tapering was dominant (led to QALY increase and incurred fewer direct costs). The uncertainty around the cost-effectiveness analysis has been assessed using the resampling technique bootstrapping with 5000 simulations, showing that our intervention would be dominant in $97.5 \%$ of our simulations. Our observations in terms of HRQoL, HCRU and cost-effectiveness are aligned with those previously reported observational studies, which assessed drug costs of RA and SpA in patients in remission or LDA treated with infliximab, adalimumab and etanercept; a decrease in drugs used which translated into reduced direct costs was observed in a Spanish University Hospital [30]. Krieckaert et al. developed a Markov model based on a 272 RA patient cohort in The Netherlands starting adalimumab therapy; patients treated following a personalised TDM strategy presented with lower disease activity and progression of functional disability based on HAQ and the intervention led to reduced costs and resulted in increased QALYs (dominant) in $72 \%$ of the simulations [21]. Similar cost-saving results were observed when using TDM for RA patients under infliximab and adalimumab treatment in Finland [22].

One of the main strengths of our study is the fact that it has been implemented through a pragmatic trial approach and therefore should be representative of usual clinical practice. Randomized controlled trials are the gold standard to assess efficacy and safety of interventions, but are not the ideal scenario to generate effectiveness, HRQoL or HCRU under routine clinical conditions. In this context, pragmatic trials, using standard of care as a comparator and leading to high external validity, are a suitable approach to generate health economic data, particularly when assessing the cost-effectiveness of medical devices such as diagnostic tests [31].

However, limitations of the study need to be acknowledged. First, the fact that the primary endpoint was not met conditions the interpretation of the rest of the study results. Additionally, although we believe the pragmatic trial approach is suitable for this type of evaluation, this type of study may have limited internal validity [31] and further studies with longer follow-up would be required. There was a certain imbalance in the percentage of patients of each rheumatological disease as well as between those in clinical remission and LDA between the CG and the IG, which were not adjusted when assessing the clinical outcomes. However, no significant differences were observed in any clinical variables between the CG and the IG at baseline, except for the disease duration for the RA and PsA populations, but this did not translate into differences in any of the other variables assessed including EQ-5D-5L utilities. In addition, costs of adverse events were not collected through the study follow-up. Also, at the time the study was conducted, no adalimumab biosimilars were available and, therefore, total drug costs considered herein may not be representative of the current costs. Finally, it should be considered that all study variables in this study reflect the situation in Spain and might not be directly applied to other health systems.

Despite knowing that serum anti-TNF concentrations would help to improve management in patients with rheumatic diseases, current clinical practices in rheumatology do not generally include routine drug monitoring [4]. In light of the increasing demand in antiTNF therapies for rheumatological conditions and for other therapeutic areas, which is increasing health expenditure, and also considering the high pressure on health policy makers in terms of limited resources, we believe that evidence that can support the efficient use of such expensive resources is extremely valuable. The present study clearly indicates for the first time that using a TDM-based strategy is economically justified, is efficient compared to the CG and would help physicians to improve healthcare resources. Our analysis revealed overall significant differences in costs, especially in adalimumab administration costs. In addition, our study would be of interest for other disease indications where adalimumab and 
other anti-TNFs agents are proven to be costeffective [28].

\section{CONCLUSIONS}

In conclusion, our results indicated that adalimumab dose tapering based on TDM-guided management in clinically stable rheumatic patients could be an efficient strategy, improving patients' HRQoL while incurring lower costs.

\section{ACKNOWLEDGMENTS}

The authors thank all the patients who contributed to this study.

Funding. This work was supported by the Spanish Ministry of Economy and Competitiveness through the INNPACTO program [IPT2012-0062-010000]. Rapid Service Fee was provided by Grifols.

Medical Writing, Editorial and other Assistance. We thank the Biobanco Vasco (Barakaldo, Spain) for sample aliquoting, storing and shipping. We also thank Eugenio Rosado, PhD, Jordi Bozzo, PhD CMPP (Grifols), and MaryJane Silvey, MBA (WriteMonitor, LLC, Durham, NC, USA) for medical writing and editorial support in the preparation of this manuscript. Elisabet Viayna (Grifols) is acknowledged for providing her expert insight to improve this manuscript.

Authorship. All named authors meet the International Committee of Medical Journal Editors (ICMJE) criteria for authorship for this article, take responsibility for the integrity of the work as a whole, and have given their approval for this version to be published.

Authors' Contributions. Catalina Gómez Arango, Eduardo Úcar, Maria Luz García-Vivar, Clara Eugenia Pérez, Juan Ramon De Dios, Belen Alvarez, Ana Ruibal Escribano, Claudia Stoye, Margarida Vasques and Joaquin Belzunegui contributed to study design, patient recruitment and follow-up, data acquisition and manuscript review for important intellectual content. Inigo Gorostiza, Antonio Escobar, Daniel Nagore, Antonio Martínez, Ziortza Trancho and Eli Díez contributed to study design, coordination, statistical data analysis, results interpretation, manuscript writing and critical review for important intellectual content. Ainhoa Ruiz del Agua, Lorena Del Rio and Cristina Jorquera contributed to sample processing and banking, analysis, results interpretation and critical review for important intellectual content.

Prior Presentation. Preliminary partial results of this study were previously presented at the European League Against Rheumatism (EULAR) 2017 Annual Meeting-Ann Rheum Dis 2017;76(2):826 and at the American College of Rheumatology/Association for Rheumatology Health Professionals (ACR/ARHP) 2017 Annual Meeting-Arthritis Rheumatol 2017;69(suppl 10).

Disclosures. Ainhoa Ruiz del Agua, Lorena Del Rio, Eli Diez, Antonio Martínez and Daniel Nagore are full-time employees of Progenika Biopharma, S.A., a Grifols company. Catalina Gómez Arango, Inigo Gorostiza, Eduardo Úcar, Maria Luz García-Vivar, Clara Eugenia Perez, Juan Ramon De Dios, Belen Alvarez, Ana Ruibal Escribano, Claudia Stoye, Margarida Vasques, Joaquin Belzunegui, Antonio Escobar, Ziortza Trancho and Cristina Jorquera declare that they have nothing to disclosure, which might be perceived as posing a conflict or bias regarding this manuscript.

Compliance with Ethics Guidelines. Each patient signed a written informed consent form before participating in the study. The study was approved by the Basque Clinical Investigation Ethics Committee and was conducted in accordance with local regulations and with the ethical principles of the current Declaration of Helsinki.

Data Availability. All data generated or analysed during this study are included in this published article/as supplementary information files. 
Open Access. This article is licensed under a Creative Commons Attribution-NonCommercial 4.0 International License, which permits any non-commercial use, sharing, adaptation, distribution and reproduction in any medium or format, as long as you give appropriate credit to the original author(s) and the source, provide a link to the Creative Commons licence, and indicate if changes were made. The images or other third party material in this article are included in the article's Creative Commons licence, unless indicated otherwise in a credit line to the material. If material is not included in the article's Creative Commons licence and your intended use is not permitted by statutory regulation or exceeds the permitted use, you will need to obtain permission directly from the copyright holder. To view a copy of this licence, visit http://creativecommons.org/licenses/by$\mathrm{nc} / 4.0 /$.

\section{REFERENCES}

1. Aletaha D, Neogi T, Silman AJ, et al. 2010 Rheumatoid arthritis classification criteria: an American College of Rheumatology/European League Against Rheumatism collaborative initiative. Arthritis Rheum. 2010;62:2569-81.

2. Coates LC, Tillett W, Chandler D, et al. The 2012 BSR and BHPR guideline for the treatment of psoriatic arthritis with biologics. Rheumatology (Oxford). 2013;52:1754-7.

3. Chiu YM, Lai MS, Lin HY, Lang HC, Lee LJ, Wang JD. Disease activity affects all domains of quality of life in patients with rheumatoid arthritis and is modified by disease duration. Clin Exp Rheumatol. 2014;32:898-903.

4. Tornero Molina J, Sanmarti Sala R, Rodriguez Valverde $\mathrm{V}$, et al. Update of the Consensus Statement of the Spanish Society of Rheumatology on the management of biologic therapies in rheumatoid arthritis. Reumatol Clin. 2010;6:23-36.

5. Vincent FB, Morand EF, Murphy K, Mackay F, Mariette X, Marcelli C. Antidrug antibodies (ADAb) to tumour necrosis factor (TNF)-specific neutralising agents in chronic inflammatory diseases: a real issue, a clinical perspective. Ann Rheum Dis. 2013;72:165-78.
6. van Kuijk AW, de Groot M, Stapel SO, Dijkmans BA, Wolbink GJ, Tak PP. Relationship between the clinical response to adalimumab treatment and serum levels of adalimumab and anti-adalimumab antibodies in patients with psoriatic arthritis. Ann Rheum Dis. 2010;69:624-5.

7. Krieckaert CL, Jamnitski A, Nurmohamed MT, Kostense PJ, Boers M, Wolbink G. Comparison of long-term clinical outcome with etanercept treatment and adalimumab treatment of rheumatoid arthritis with respect to immunogenicity. Arthritis Rheum. 2012;64:3850-5.

8. Bartelds GM, Krieckaert CL, Nurmohamed MT, et al. Development of antidrug antibodies against adalimumab and association with disease activity and treatment failure during long-term follow-up. JAMA. 2011;305:1460-8.

9. Bojke L, Epstein D, Craig D, et al. Modelling the cost-effectiveness of biologic treatments for psoriatic arthritis. Rheumatology (Oxford). 2011;50(Suppl 4):iv39-47.

10. Kobelt G, Sobocki P, Mulero J, Gratacos J, Pocovi A, Collantes-Estevez E. The burden of ankylosing spondylitis in Spain. Value Health. 2008;11:408-15.

11. Rodgers M, Epstein D, Bojke L, et al. Etanercept, infliximab and adalimumab for the treatment of psoriatic arthritis: a systematic review and economic evaluation. Health Technol Assess. 2011;15: $1-329$.

12. Wailoo AJ, Bansback N, Brennan A, Michaud K, Nixon RM, Wolfe F. Biologic drugs for rheumatoid arthritis in the Medicare program: a cost-effectiveness analysis. Arthritis Rheum. 2008;58:939-46.

13. Dreesen E, Bossuyt P, Mulleman D, Gils A, PascualSalcedo D. Practical recommendations for the use of therapeutic drug monitoring of biopharmaceuticals in inflammatory diseases. Clin Pharmacol. 2017;9: 101-11.

14. Bartelds GM, Wijbrandts CA, Nurmohamed MT, et al. Clinical response to adalimumab: relationship to anti-adalimumab antibodies and serum adalimumab concentrations in rheumatoid arthritis. Ann Rheum Dis. 2007;66:921-6.

15. Pouw MF, Krieckaert CL, Nurmohamed MT, et al. Key findings towards optimising adalimumab treatment: the concentration-effect curve. Ann Rheum Dis. 2015;74:513-8.

16. den Broeder AA, van der Maas A, van den Bemt BJ. Dose de-escalation strategies and role of therapeutic drug monitoring of biologics in RA. Rheumatology (Oxford). 2010;49:1801-3. 
17. Arends S, van der Veer E, Kamps FB, et al. Patienttailored dose reduction of TNF-alpha blocking agents in ankylosing spondylitis patients with stable low disease activity in daily clinical practice. Clin Exp Rheumatol. 2015;33:174-80.

18. Marotte H, Rinaudo-Gaujous M, Petiet C, Fautrel B, Paul S. Tapering without relapse in rheumatoid arthritis patients with high TNF blocker concentrations: data from STRASS study. Ann Rheum Dis. 2020;79:081.

19. Gonzalez-Alvaro I, Martinez-Fernandez C, Dorantes-Calderon B, et al. Spanish Rheumatology Society and Hospital Pharmacy Society Consensus on recommendations for biologics optimization in patients with rheumatoid arthritis, ankylosing spondylitis and psoriatic arthritis. Rheumatology (Oxford). 2015;54:1200-9.

20. de la Torre I, Valor L, Nieto JC, et al. Anti-TNF treatments in rheumatoid arthritis: economic impact of dosage modification. Expert Rev Pharmacoecon Outcomes Res. 2013;13:407-14.

21. Krieckaert CL, Nair SC, Nurmohamed MT, et al. Personalised treatment using serum drug levels of adalimumab in patients with rheumatoid arthritis: an evaluation of costs and effects. Ann Rheum Dis. 2015;74:361-8.

22. Laine J, Jokiranta TS, Eklund KK, Vakevainen M, Puolakka K. Cost-effectiveness of routine measuring of serum drug concentrations and anti-drug antibodies in treatment of rheumatoid arthritis patients with TNF-alpha blockers. Biologics. 2016;10:67-73.

23. Rabin R, de Charro F. EQ-5D: a measure of health status from the EuroQol Group. Ann Med. 2001;33: 337-43.

24. den Broeder AA, van Herwaarden N, van der Maas $A$, et al. Dose REduction strategy of subcutaneous
TNF inhibitors in rheumatoid arthritis: design of a pragmatic randomised non inferiority trial, the DRESS study. BMC Musculoskelet Disord. 2013;14: 299.

25. Ramos-Goñi JM, Craig BM, Oppe M, et al. Handling data quality issues to estimate the Spanish EQ-5D$5 \mathrm{~L}$ value set using a hybrid interval regression approach. Value Health. 2018;21:596-604.

26. Vallejo-Torres L, Garcia-Lorenzo B, Serrano-Aguilar P. Estimating a cost-effectiveness threshold for the Spanish NHS. Health Econ. 2018;27:746-61.

27. I'Ami MJ, Krieckaert CL, Nurmohamed MT, et al. Successful reduction of overexposure in patients with rheumatoid arthritis with high serum adalimumab concentrations: an open-label non-inferiority, randomised clinical trial. Ann Rheum Dis. 2018;77:484-7.

28. Martelli L, Olivera P, Roblin X, Attar A, Peyrin-Biroulet L. Cost-effectiveness of drug monitoring of anti-TNF therapy in inflammatory bowel disease and rheumatoid arthritis: a systematic review. J Gastroenterol. 2017;52:19-25.

29. Lamot L, Bukovac LT, Vidovic M, Frleta M, Harjacek M. The "head-to-head" comparison of etanercept and infliximab in treating children with juvenile idiopathic arthritis. Clin Exp Rheumatol. 2011;29: 131-9.

30. Pascual-Salcedo DP, Plasencia C, del Gonzalez Valle L. THU0189 Therapeutic drug monitoring (TDM) in rheumatic day clinic enables to reduce pharmaceutical cost maintaining clinical efficacy. Ann Rheum Dis. 2013;72(Suppl 3):A227.

31. Marshall DA, Hux M. Design and analysis issues for economic analysis alongside clinical trials. Med Care. 2009;47:S14-20. 